\title{
CD90- (Thy-1-) High Selection Enhances Reprogramming Capacity of Murine Adipose-Derived Mesenchymal Stem Cells
}

\author{
Koichi Kawamoto, ${ }^{1}$ Masamitsu Konno, ${ }^{2}$ Hiroaki Nagano, ${ }^{2}$ Shimpei Nishikawa, \\ Yoshito Tomimaru, ${ }^{2}$ Hirofumi Akita, ${ }^{2}$ Naoki Hama, ${ }^{2}$ Hiroshi Wada, ${ }^{2}$ Shogo Kobayashi, ${ }^{2}$ \\ Hidetoshi Eguchi, ${ }^{2}$ Masahiro Tanemura, ${ }^{3}$ Toshinori Ito, ${ }^{4}$ Yuichiro Doki, ${ }^{1}$ Masaki Mori, \\ and Hideshi Ishii ${ }^{2}$ \\ ${ }^{1}$ Department of Surgery, Graduate School of Medicine, Osaka University, 2-2 Yamadaoka, Suita, Osaka 565-0871, Japan \\ ${ }^{2}$ Department of Frontier Science for Cancer and Chemotherapy, Graduate School of Medicine, Osaka University, 2-2 Yamadaoka, \\ Suita, Osaka 565-0871, Japan \\ ${ }^{3}$ Department of Surgery and Institute for Clinical Research, National Hospital Organization Kure Medical Center and Chugoku Cancer \\ Center, Hiroshima 737-0023, Japan \\ ${ }^{4}$ Department of Complementary and Alternative Medicine, Graduate School of Medicine, Osaka University, 2-2 Yamadaoka, Suita, \\ Osaka 565-0871, Japan
}

Correspondence should be addressed to Hiroaki Nagano; hnagano@gesurg.med.osaka-u.ac.jp and Hideshi Ishii; hishii@gesurg.med.osaka-u.ac.jp

Received 18 June 2013; Revised 7 September 2013; Accepted 12 September 2013

Academic Editor: Chao Hung Hung

Copyright ( 2013 Koichi Kawamoto et al. This is an open access article distributed under the Creative Commons Attribution License, which permits unrestricted use, distribution, and reproduction in any medium, provided the original work is properly cited.

Background. Mesenchymal stem cells (MSCs), including adipose tissue-derived mesenchymal stem cells (ADSC), are multipotent and can differentiate into various cell types possessing unique immunomodulatory features. Several clinical trials have demonstrated the safety and possible efficacy of MSCs in organ transplantation. Thus, stem cell therapy is promising for tolerance induction. In this study, we assessed the reprogramming capacity of murine ADSCs and found that CD90 (Thy-1), originally discovered as a thymocyte antigen, could be a useful marker for cell therapy. Method. Murine ADSCs were isolated from B6 mice, sorted using a FACSAria cell sorter by selection of $\mathrm{CD} 90^{\mathrm{Hi}}$ or $\mathrm{CD} 90^{\mathrm{Lo}}$, and then transduced with four standard factors (4F; Oct4, Sox2, Klf4, and c-Myc). Results. Unsorted, CD90 ${ }^{\mathrm{Hi}}$-sorted, and $\mathrm{CD} 90^{\mathrm{Lo}}$-sorted murine ADSCs were reprogrammed using standard $4 \mathrm{~F}$ transduction. $\mathrm{CD} 90^{\mathrm{Hi}}$ ADSCs showed increased numbers of alkaline phosphatase-positive colonies compared with $\mathrm{CD} 90^{\mathrm{Lo}}$ ADSCs. The relative reprogramming efficiencies of unsorted, $\mathrm{CD} 90^{\mathrm{Hi}}$-sorted, and $\mathrm{CD} 90^{\mathrm{Lo}}$-sorted ADSCs were $100 \%, 116.5 \%$, and $74.7 \%$, respectively. $\mathrm{CD} 90^{\mathrm{Hi}}$ cells were more responsive to reprogramming. Conclusion. $\mathrm{CD} 90^{\mathrm{Hi}}$ ADSCs had greater reprogramming capacity than $\mathrm{CD}^{2} 0^{\mathrm{Lo}}$ ADSCs, suggesting that ADSCs have heterogeneous subpopulations. Thus, CD90 ${ }^{\mathrm{Hi}}$ selection presents an effective strategy to isolate a highly suppressive subpopulation for stem cell-based tolerance induction therapy.

\section{Introduction}

Induced pluripotent stem (iPS) cells can be directly generated from fibroblast cultures by expression of four factors $(4 \mathrm{~F})$, including octamer-binding transcription factor 4 , sexdetermining region Y-box 2, Kruppel-like factor 4, and myelocytomatosis viral oncogene homolog [1]. Although these factors present attractive sources for stem cell therapy, the mechanisms by which they are generated are not fully understood. The inefficiency of iPS cell generation has prompted the development of two contending models, namely, the stochastic and elite models. A recent multilineage-differentiating stress-enduring cell study suggested the greater utility of the elite model over the stochastic model [2]. In either model, stem cells or progenitor cells isolated for experimental or therapeutic research are usually heterogeneous populations; therefore, a more favorable subfraction for use in stem cell therapy may 
exist. Indeed, several reports have suggested that the differentiation stage of the starting cell line has a critical influence on the efficiency of reprogramming into iPS cells in hematopoiesis [3]. Immature cell populations, such as inn $^{-} \mathrm{c}-\mathrm{Kit}^{+} \mathrm{Scal}{ }^{+} \mathrm{CD} 48^{-} \mathrm{CD} 150^{+} \mathrm{CD} 34^{-}$(lack of lineage/Hardy-Zuckerman 4 feline sarcoma viral oncogene homolog/spinocerebellar ataxia/cluster of differentiation $48^{-} / 150^{+} / 34^{-}$) hematopoietic stem cells, in general give rise to iPS cells at higher efficiencies than terminally differentiated cell types. Furthermore, a recent study demonstrated that $\mathrm{CD} 90^{\mathrm{Hi}}$ adipose tissue-derived mesenchymal stem cells (ADSCs) are more capable of forming bone both in vitro and in vivo than in $\mathrm{CD}_{105}{ }^{\mathrm{Lo}}$ cells [4]. CD90 (Thy-1) is a 25$37-\mathrm{kDa}$ glycosylphosphatidylinositol-anchored glycoprotein expressed mainly in leukocytes that have been used as murine pan T-cell markers, like CD2, CD5, and CD28.

Multipotent mesenchymal stem cells (MSCs), which represent a nonhematopoietic cell population, can differentiate into mesenchymal tissues (i.e., bone, cartilage, or fat) $[5,6]$; they were first isolated from bone marrow and later from nonmarrow tissues, including umbilical cord blood and adipose tissue $[5,6]$. Immunologically, MCSs are known to be less immunogenic because of, at least in part, the lack of surfaceexpressed human leukocyte antigens [5]. Moreover, they have been shown to possess anti-inflammatory and immunosuppressive effects both in murine and human models of diabetes $[7,8]$. Thus, the immunoregulatory properties of MSCs are appealing, because they inhibit T-cell proliferation and differentiation of monocytes to dendritic cells, modulate B-cell functions, and suppress natural killer cell cytotoxicity. Solid organ transplantation is an established and useful treatment option for end-stage organ failure. Despite excellent shortterm results, long-term survival of transplanted grafts has not improved accordingly because of graft rejection, graft fibrosis, or side effects of immunosuppressants. The aim of stem cell therapy for solid organ transplantation is to prevent or treat acute rejection and to involve autologous or allogeneic stem cell transplantation into patients, either through local delivery or systemic infusion. Currently, there are over 100 ongoing clinical trials to evaluate the utility of MSCs, especially in kidney transplantation [9-11].

ADSCs have been shown to consist of heterogeneous subpopulations; therefore, we hypothesized that $\mathrm{CD} 90^{\mathrm{Hi}} \mathrm{ADSCs}$ might exhibit increased reprogramming capacities and subsequently have better immunoregulatory effects. Hence, the aim of the present study was to compare the reprogramming capacity of sorted murine $\mathrm{CD} 90^{\mathrm{Hi}}$ ADSCs. Our data demonstrated that $\mathrm{CD} 90^{\mathrm{Hi}}$ selection improved the reprogramming capacity of murine ADSCs, suggesting that $\mathrm{CD} 90^{\mathrm{Hi}}$ ADSCs could help prevent graft rejection.

\section{Materials and Methods}

2.1. ADSC Isolation. Murine ADSCs were isolated from $\mathrm{B} 6$ mice as previously described but with slight modifications [7]. Briefly, adipose tissue was obtained from the inguinal fat pads, washed with Dulbecco's phosphate-buffered saline (DPBS; Invitrogen, Carlsbad, CA, USA) containing $50 \mathrm{U} / \mathrm{mL}$ of

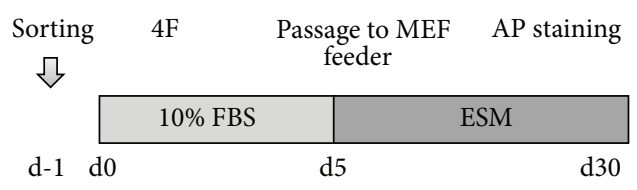

FIGURE 1: Lentiviral-mediated transfer of four iPS cell factor genes in murine ADSCs. A schematic representation of the experiment is shown. Cells were transduced with four factors (4F) after $24 \mathrm{~h}$ of incubation. Then, $4 \mathrm{~F}$-transduced cells were passaged to MMCtreated MEF feeder cells on day 5 . The number of reprogrammed iPS colonies was assessed on posttransduction day 30 by AP staining.

penicillin and $50 \mu \mathrm{g} / \mathrm{mL}$ of streptomycin (PSM; Invitrogen), and then cut into fine pieces, which were incubated with D-PBS containing $1.0 \mathrm{mg} / \mathrm{mL}$ of Clostridium histolyticum collagenase (Sigma-Aldrich, St. Louis, MO, USA) in a $37^{\circ} \mathrm{C}$ shaking incubator for $1 \mathrm{~h}$. The digested tissue was filtered through sterile $70-\mu \mathrm{m}$ nylon mesh, centrifuged at $430 \times \mathrm{g}$ for $5 \mathrm{~min}$, and resuspended in Dulbecco's modified Eagle's medium (DMEM) (Nacalai Tesque, Kyoto, Japan) supplemented with $10 \%$ fetal bovine serum (FBS; HyClone; Thermo Scientific, Waltham, MA, USA) and PSM.

2.2. Cell Sorting. Murine ADSCs were washed with D-PBS and treated with Accutase reagent (EMD Millipore, Billerica, MA, USA) in a $37^{\circ} \mathrm{C}$ incubator for $2-3 \mathrm{~min}$ to dissociate the cells, which were then resuspended in staining media [D-PBS supplemented with FBS (1\%) and EDTA (2 mM)]. Next, the cells were stained with fluorescein isothiocyanate-conjugated anti-mouse CD34 (eBioscience, San Diego, CA, USA), phycoerythrin-conjugated anti-mouse CD31, eFluor450 antimouse CD45, and allophycocyanin-conjugated anti-mouse CD90.2 for $60 \mathrm{~min}$ at $4^{\circ} \mathrm{C}$. The cells were then washed with staining media, stained with 7-aminoactinomycin D to exclude dead cells, and sorted using the FACSAria cell sorter (Bio-Rad, Hercules, CA, USA) [12]. The particular phenotypic subsets of $\mathrm{CD} 90^{\mathrm{Hi}}$ and CD90 $0^{\mathrm{Lo}}$ ADSCs, based on CD31, CD45, and CD90 expression, were evaluated by multicolor analysis and sorted by adequate gating.

2.3. Lentiviral Preparation. To prepare the lentiviruses, human embryonic kidney (HEK)-293Ta cells were cultured in DMEM supplemented with 10\% FBS and PSM. Then, the cells were seeded at $5.0 \times 10^{6}$ cells per $100 \mathrm{~mm}$ dish 1 day before viral transduction. Fugene 6 transfection reagent (22.5 $\mu \mathrm{L}$; Promega, Madison, WI, USA) was diluted with $500 \mu \mathrm{L}$ of DMEM and incubated for $5 \mathrm{~min}$ at room temperature. Plasmid DNA $(2.5 \mu \mathrm{g})$ was added to the mixture, which was incubated for an additional $15 \mathrm{~min}$ at room temperature. Then, the culture media was replaced with fresh DMEM supplemented 10\% FBS and the DNA/Fugene 6 mixture was added dropwise onto the HEK-293Ta cells. The medium was replaced after $24 \mathrm{~h}$. After an additional $48 \mathrm{~h}$, virus-containing supernatants, derived from the HEK-293Ta cultures, were filtered through a $0.22-\mu \mathrm{L}$ cellulose-acetate filter and analyzed without being frozen. 


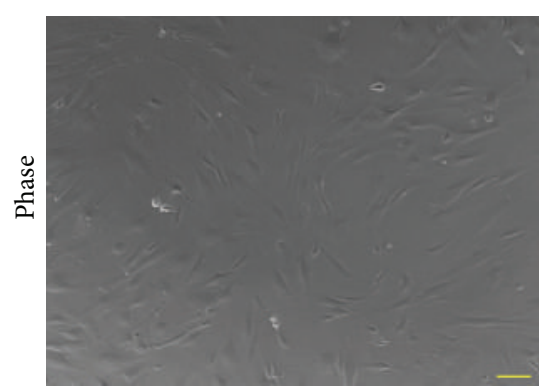

(a)

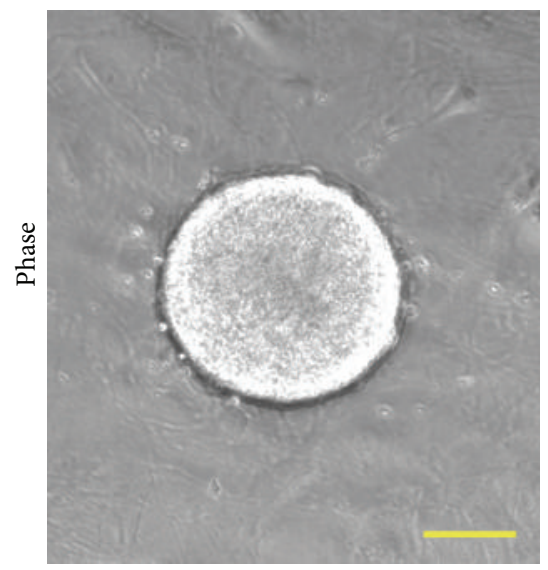

(c)

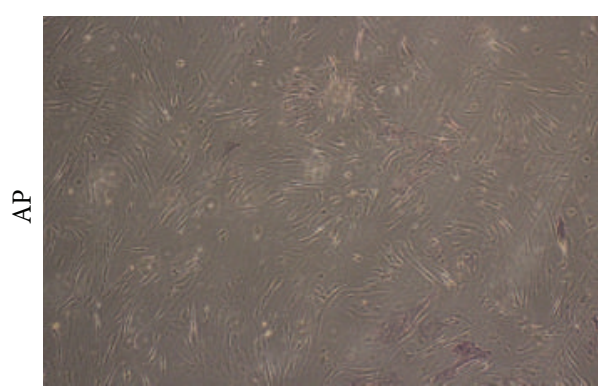

(b)

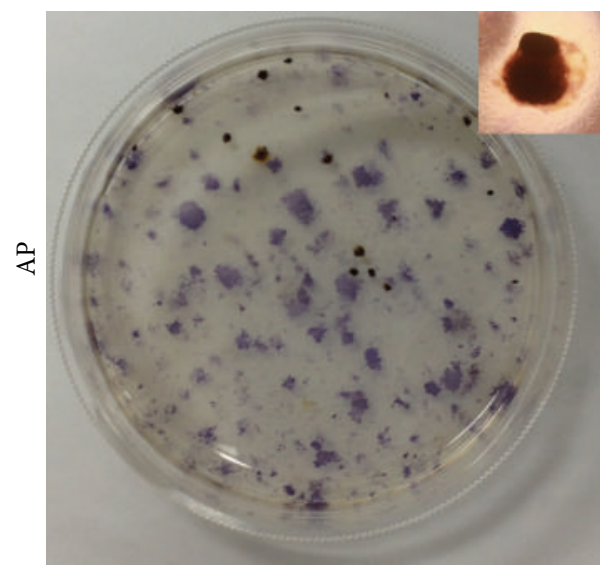

(d)

FIgure 2: 4F transduction resulted in iPS colony formation in unsorted murine ADSCs. Phase (a and c) and AP staining (b and d) of preand post- (c and $d$ ) transduction were shown.

2.4. Lentiviral Transduction. Sorted $\mathrm{CD} 90^{\mathrm{Hi}}$ and $\mathrm{CD} 90^{\mathrm{Lo}}$ cells were plated onto 6-well culture plates at a density of $1.0 \times 10^{5}$ cells/well, respectively. After incubation for $24 \mathrm{~h}$, the culture medium was retrieved and viral supernatants were added to each well. The next day, the cells were washed with complete medium three times. Mouse embryonic fibroblasts (MEFs) were mitotically inactivated by the addition of $10 \mu \mathrm{g} / \mathrm{mL}$ of mitomycin C (MMC; Nacalai Tesque Inc., Kyoto, Japan) for $90 \mathrm{~min}$ and then washed with D-PBS three times. The cells were then cultured with complete medium for at least $2 \mathrm{~h}$, trypsinized, counted, and plated on gelatincoated $60 \mathrm{~mm}$ dishes at a density of $5.0 \times 10^{5}$ cells/well. On posttransduction day 5 , the cells were trypsinized; suspended in DMEM supplemented with 15\% (v/v) FBS, PSM, 1-mM sodium pyruvate (Invitrogen), $10^{-4} \mathrm{M}$ 2-mercaptoethanol (Nacalai Tesque, Inc.), nonessential amino acid solution, and $1,000 \mathrm{U} / \mathrm{mL}$ of leukemia inhibitory factor; after which they were counted. Then, 1000 cells were transferred to MMCtreated MEF feeder cells. On post-transduction day 30, alkaline phosphatase (AP) staining was performed to assess reprogramming efficiency.

2.5. AP Staining. 4F-transduced ADSCs were stained using an AP staining kit (Muto Pure Chemicals, Tokyo, Japan) following the manufacturer's recommended protocol. Briefly, cultured cells were rinsed twice with D-PBS and then fixed for $5 \mathrm{~s}$ in ice-cold methanol. Then, the cells were washed with tap water, stained with AP solution at $37^{\circ}$ for $120 \mathrm{~min}$, and washed again with running water. The number of $\mathrm{AP}^{+}$colonies were manually inspected and counted.

\section{Results}

Figure 1 describes the experimental protocol used in this study. First, we evaluated the reprogramming capacity of unsorted ADSCs. As shown in Figure 2, parental ADSC showed no colonies and AP staining was negative (negative control). However, $4 \mathrm{~F}$ transduction resulted in ADSC colony formation. After culturing for 30 days, the cells were stained with AP to test iPS cell pluripotency. AP staining was positive in all colonies. These results clearly demonstrated that murine ADSCs can be reprogrammed using standard 4F. Next we used sorted ADSCs to assess the reprogramming capacities of $\mathrm{CD} 90^{\mathrm{Hi}}$ and $\mathrm{CD} 90^{\mathrm{Low}}$ ADSCs. As shown in Figure 3(a), the CD90 marker was widely expressed on the surfaces of the $\mathrm{CD} 31^{-} \mathrm{CD} 45^{-} \mathrm{CD} 34^{-}$ADSCs. After $24 \mathrm{~h}$ of incubation, sorted $\mathrm{CD} 90^{\mathrm{Hi}}$ or $\mathrm{CD} 90^{\mathrm{Lo}}$ ADSCs became attached to the culture dishes (Figure 3(b)) and both showed similar morphologies. Next, we assessed the effect of in vitro culture. As shown in Figure 4(a), 7-day culture resulted in the reexpression of CD90 even after sorted CD $90^{\mathrm{Lo}}$ ADSCs. These results suggest that sorted cells might be transduced as soon as possible.

To show the successful induction of the undifferentiated state, we employed immunocytochemistry of Oct 4 


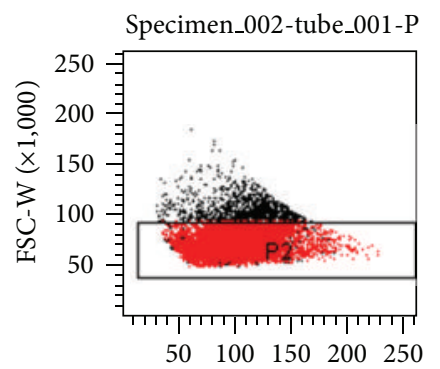

FSC-H $(\times 1,000)$

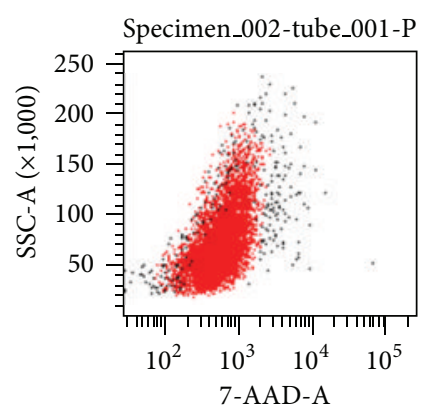

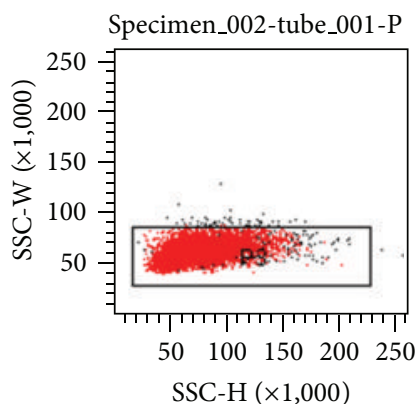

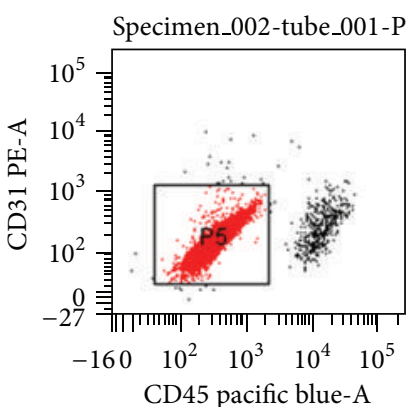

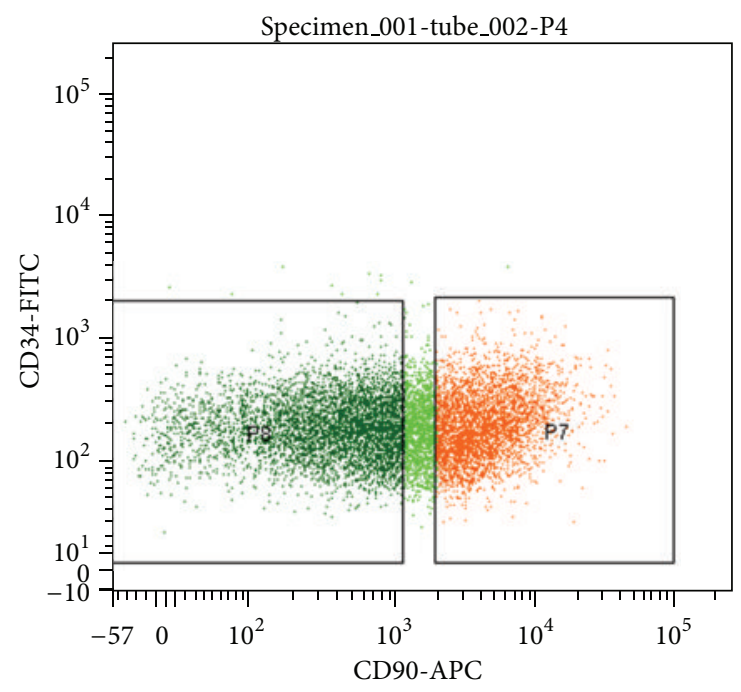

(a)
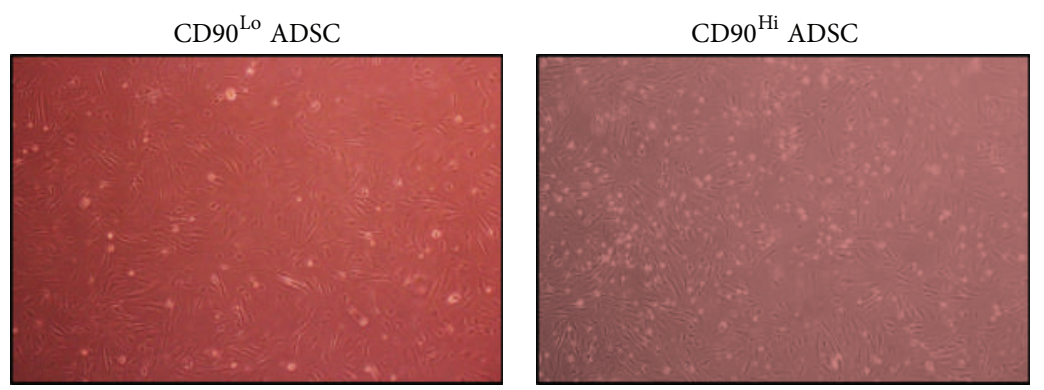

(b)

FIGURE 3: CD90 ${ }^{\mathrm{Hi}}$ and CD90 ${ }^{\mathrm{Lo}}$ sorting. (a) Gates for $\mathrm{CD} 90^{\mathrm{Hi}}$ (P7) and CD90 ${ }^{\mathrm{Lo}}$ (P8) are shown. (b) Sorted cells showed similar morphologies $24 \mathrm{~h}$ after sorting. Then, the cells were transduced with $4 \mathrm{~F}$.

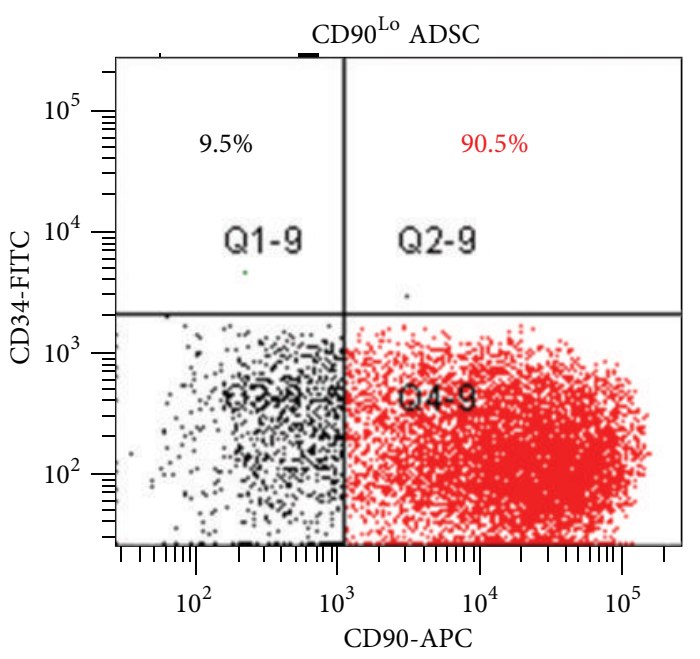

(a)

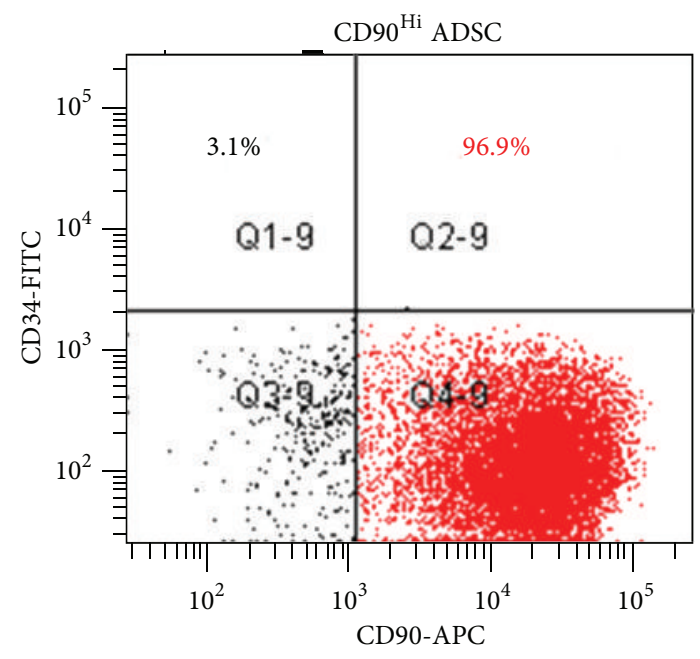

(b)

FIgURE 4: Phenotypical analysis after in vitro culture of sorted ADSCs. 

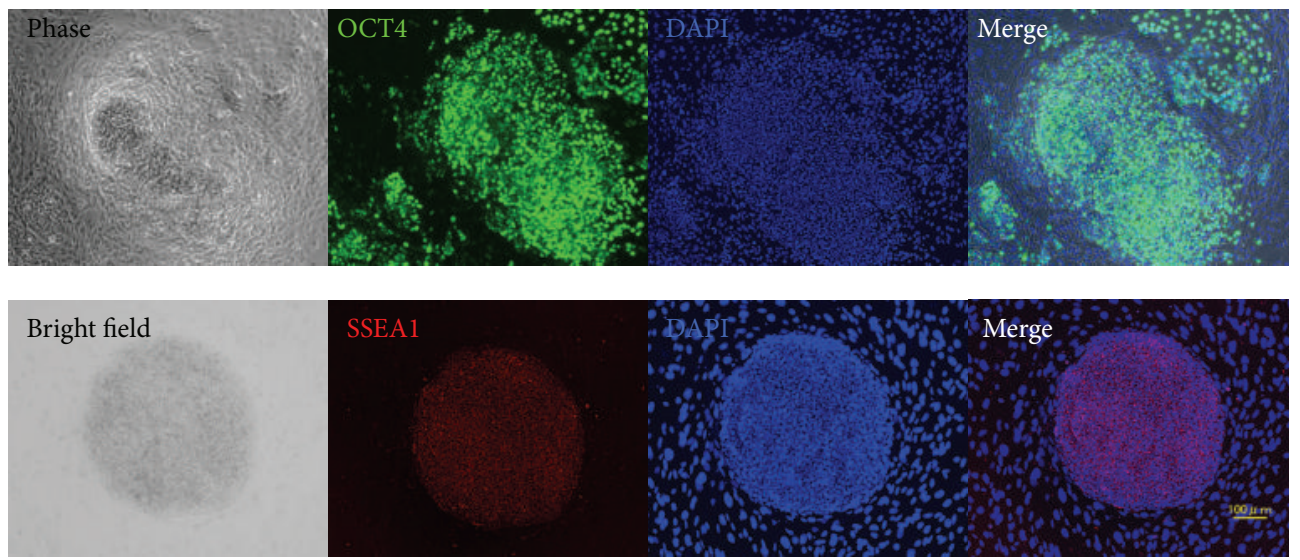

FIGURE 5: Immunocytochemistry of iPS colonies.

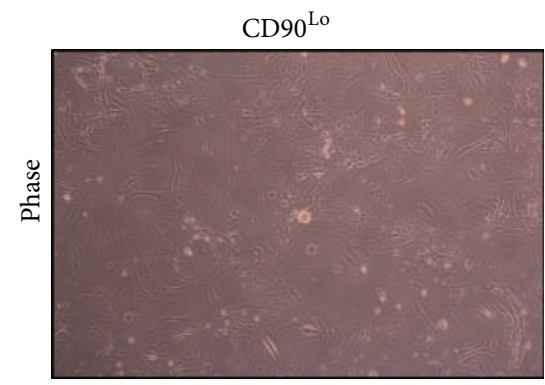

(a)

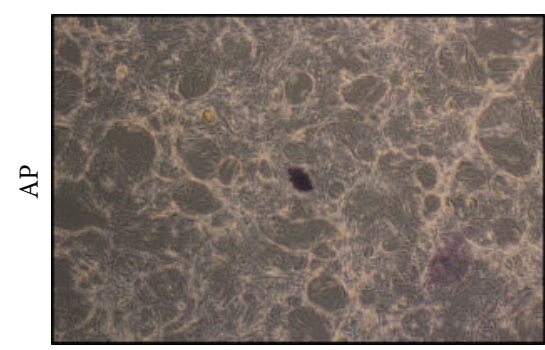

(c)

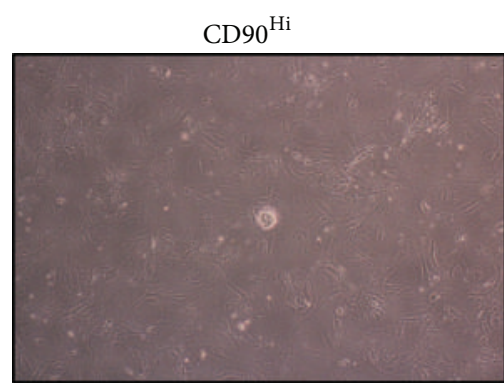

(b)

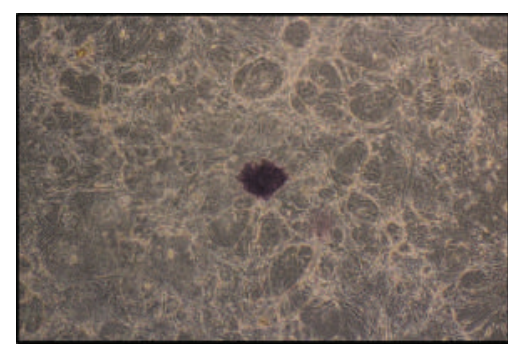

(d)

FIGURE 6: Colony formation of the sorted cells. Phase ( $\mathrm{a}$ and b) and alkaline phosphatase staining (c and d) of CD90 ${ }^{\mathrm{Lo}}\left(\mathrm{a}\right.$ and c) and CD90 ${ }^{\mathrm{Hi}}$ (b and d) are shown.

and SSEA1. As shown in Figure 5, colonies were positive for Oct4 and SSEA1. Sorted and 4F-transduced ADSCs also exhibited colony formation. As shown in Figures 6(a) and 6(b), morphologically distinct colonies were visible in both CD $90^{\mathrm{Hi}}$ and $\mathrm{CD} 90^{\mathrm{Lo}} 4 \mathrm{~F}$-transduced ADSCs on posttransduction day 14 . On posttransduction day 30, the colonies were stained with AP. There were AP+ colonies in both groups, although the $\mathrm{CD} 90^{\mathrm{Hi}}$ ADSCs tended to form larger colonies than CD90 ${ }^{\text {Lo }}$ ADSCs (Figures 4(c) and 4(d)). As shown in Figure 7, CD90 ${ }^{\mathrm{Hi}}$ ADSCs exhibited more $\mathrm{AP}+$ colonies than CD90 ${ }^{\mathrm{Lo}}$ ADSCs. The reprogramming efficiencies of unsorted, CD90 ${ }^{\mathrm{Hi}}$-sorted, and $\mathrm{CD} 90^{\mathrm{Lo}}$-sorted ADSCs were $100 \%, 116.5 \%$, and $74.7 \%$, respectively.

\section{Discussion}

In the present study, we demonstrated that murine ADSCs can be reprogrammed by standard $4 \mathrm{~F}$ transduction. Furthermore, iPS cell formation was also observed in CD90based sorted cells and the $\mathrm{CD} 90^{\mathrm{Hi}}$ sorting resulted in enhanced reprogramming capacity of murine ADSCs compared with CD90 ${ }^{\mathrm{Lo}}$ ADSCs with regard to colony number. Moreover, there was a trend in the association between CD90 expression level and individual colony size. These results clearly demonstrated that ADSCs have heterogeneous subpopulations and that the $\mathrm{CD} 90^{\mathrm{Hi}}$ ADSCs present favorable candidates for the application of clinical stem cell therapy. 


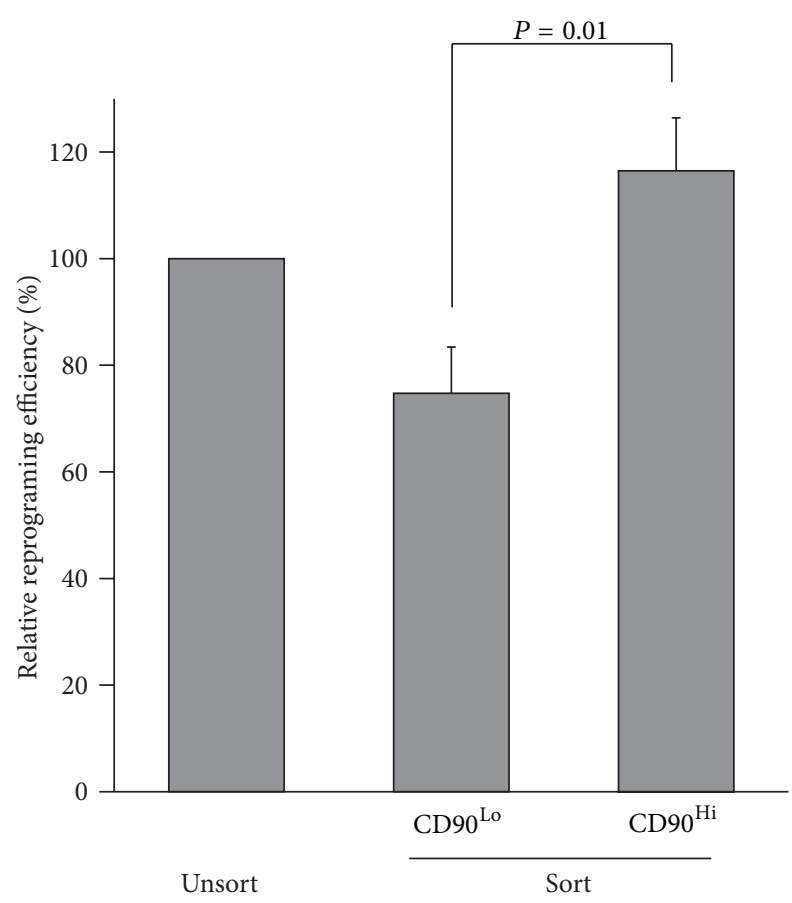

FIGURE 7: Relative reprogramming efficiency of sorted cells compared with unsorted controls.

The importance of CD90 in oncogenesis or properties of cancer stem cells has been reported [13]. CD90 expression reportedly has prognostic values in esophageal squamous cell carcinoma (ESCC) patients, because higher CD90 expression was significantly associated with a strong family history of ESCC and higher incidences of lymph node metastasis. However, the importance of CD90 expression in MSCs has not been fully elucidated; therefore, our next project is to clarify the mechanisms of CD90-mediated immunomodulatory effects. Of note, recent study reported that CD90-expressing niche stromal cells would support hematopoiesis [14].

Stem cell therapy for autoimmune disease is also well described. In a murine inflammatory bowel disease model, ADSCs alleviated experimental colitis by inhibiting inflammatory and autoimmune responses [15]. In mice, Thy-1 is also expressed by thymocytes, peripheral $\mathrm{T}$ cells, myoblasts, epidermal cells, and keratinocytes.

We and others previously reported that it is possible to reprogram murine and human cells to pluripotency by direct transfection of mature double-stranded microRNAs without viral vectors [16-18]. These viral-free strategies present effective methods for future stem cell therapy.

Currently, studies are underway to compare the immunoregulatory properties of viral-free strategies both in vitro and in vivo.

\section{Acknowledgment}

Lentiviral vectors (CSII-CMV-mOct3/4-IRES2-Venus, CSII-CMV-mSox2-IRES2-Venus, CSII-CMV-mKlf4-IRES2Venus, CSII-CMV-mcMyc-IRES2-Venus, pCAG-HIVgp, and pCAG-VSV-G-RSV-Rev) were kindly provided by the Hiroyuki Miyoshi (RIKEN; The Institute of Physical and Chemical Research, Tsukuba, Japan).

\section{References}

[1] K. Takahashi and S. Yamanaka, "Induction of pluripotent stem cells from mouse embryonic and adult fibroblast cultures by defined factors," Cell, vol. 126, no. 4, pp. 663-676, 2006.

[2] S. Wakao, M. Kitada, Y. Kuroda et al., "Multilineage-differentiating stress-enduring (Muse) cells are a primary source of induced pluripotent stem cells in human fibroblasts," Proceedings of the National Academy of Sciences of the United States of America, vol. 108, no. 24, pp. 9875-9880, 2011.

[3] S. Eminli, A. Foudi, M. Stadtfeld et al., "Differentiation stage determines potential of hematopoietic cells for reprogramming into induced pluripotent stem cells," Nature Genetics, vol. 41, no. 9, pp. 968-976, 2009.

[4] M. T. Chung, C. Liu, J. S. Hyun et al., "CD90 (Thy-1)-positive selection enhances osteogenic capacity of human adiposederived stromal cells," Tissue Engineering A, vol. 19, pp. 989-997, 2013.

[5] K. Le Blanc and D. Mougiakakos, "Multipotent mesenchymal stromal cells and the innate immune system," Nature Reviews Immunology, vol. 12, no. 5, pp. 383-396, 2012.

[6] M. Konno, A. Hamabe, S. Hasegawa et al., "Adipose-derived mesenchymal stem cells and regenerative medicine," Development, Growth and Differentiation, vol. 55, pp. 309-318, 2013.

[7] Y. Ohmura, M. Tanemura, N. Kawaguchi et al., "Combined transplantation of pancreatic islets and adipose tissue-derived stem cells enhances the survival and insulin function of islet grafts in diabetic mice," Transplantation, vol. 90, no. 12, pp. 1366-1373, 2010.

[8] T. Y. Yeung, K. L. Seeberger, T. Kin et al., "Human mesenchymal stem cells protect human islets from pro-inflammatory cytokines," PLoS ONE, vol. 7, Article ID e38189, 2012.

[9] J. Tan, W. Wu, X. Xu et al., "Induction therapy with autologous mesenchymal stem cells in living-related kidney transplants: a randomized controlled trial," Journal of the American Medical Association, vol. 307, no. 11, pp. 1169-1177, 2012.

[10] Y. Peng, M. Ke, L. Xu et al., "Donor-derived mesenchymal stem cells combined with low-dose tacrolimus prevent acute rejection after renal transplantation: a clinical pilot study," Transplantation, vol. 95, pp. 161-168, 2013.

[11] M. E. Reinders, J. W. de Fijter, H. Roelofs et al., "Autologous bone marrow-derived mesenchymal stromal cells for the treatment of allograft rejection after renal transplantation: results of a phase I study," Stem Cells Translational Medicine, vol. 2, pp. 107-111, 2013.

[12] S. Nishikawa, M. Konno, A. Hamabe et al., "Aldehyde dehydrogenase high gastric cancer stem cells are resistant to chemotherapy," International Journal of Oncology, vol. 42, pp. 1437-1442, 2013.

[13] K. H. Tang, Y. D. Dai, M. Tong et al., "A CD90(+) tumorinitiating cell population with an aggressive signature and metastatic capacity in esophageal cancer," Cancer Research, vol. 73, pp. 2322-2332, 2013.

[14] C. K. Chan, P. Lindau, W. Jiang et al., "Clonal precursor of bone, cartilage, and hematopoietic niche stromal cells," Proceedings of the National Academy of Sciences of the United States of America, vol. 110, no. 31, pp. 12643-12648, 2013. 
[15] M. A. González, E. Gonzalez-Rey, L. Rico, D. Büscher, and M. Delgado, "Adipose-derived mesenchymal stem cells alleviate experimental colitis by inhibiting inflammatory and autoimmune responses," Gastroenterology, vol. 136, no. 3, pp. 978-989, 2009.

[16] K. Okita, M. Nakagawa, H. Hyenjong, T. Ichisaka, and S. Yamanaka, "Generation of mouse induced pluripotent stem cells without viral vectors," Science, vol. 322, no. 5903, pp. 949-953, 2008.

[17] N. Miyoshi, H. Ishii, H. Nagano et al., "Reprogramming of mouse and human cells to pluripotency using mature microRNAs," Cell Stem Cell, vol. 8, no. 6, pp. 633-638, 2011.

[18] F. Anokye-Danso, C. M. Trivedi, D. Juhr et al., "Highly efficient miRNA-mediated reprogramming of mouse and human somatic cells to pluripotency," Cell Stem Cell, vol. 8, no. 4, pp. 376-388, 2011. 


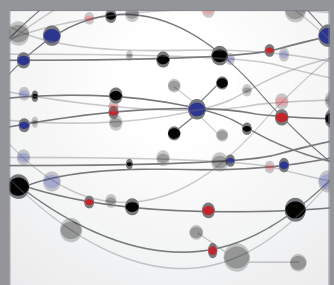

The Scientific World Journal
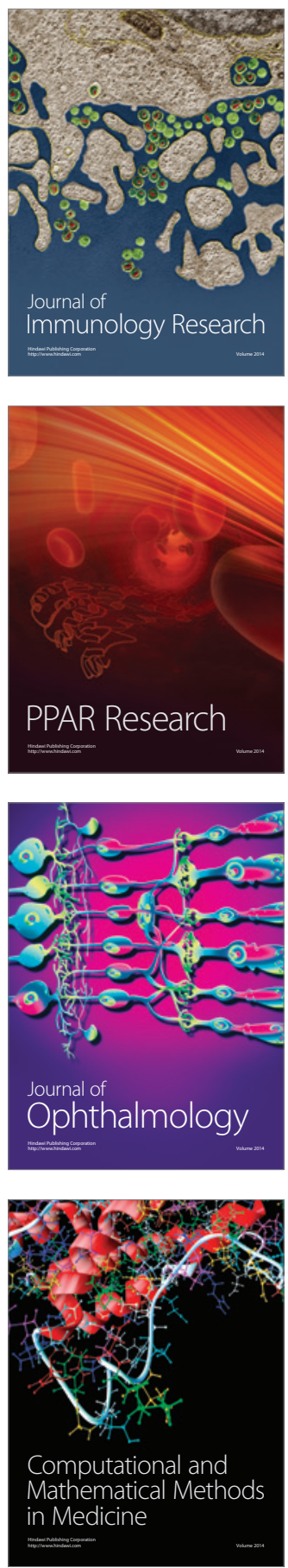

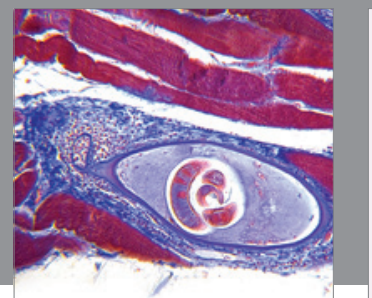

Gastroenterology

Research and Practice
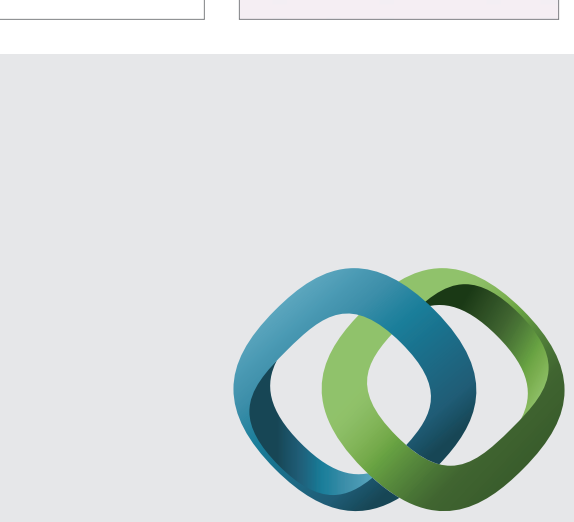

\section{Hindawi}

Submit your manuscripts at

http://www.hindawi.com
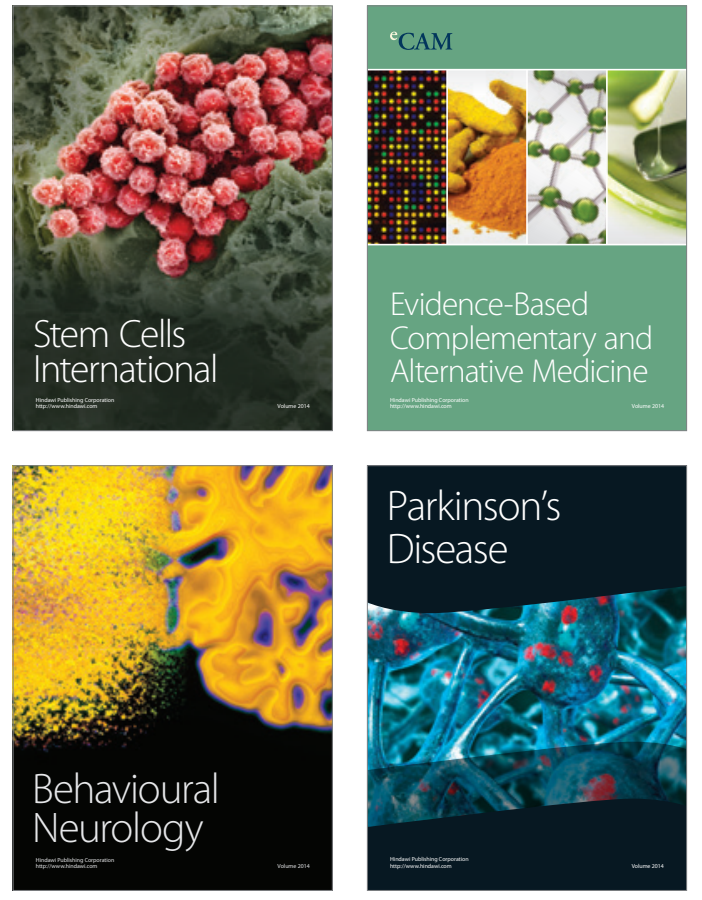
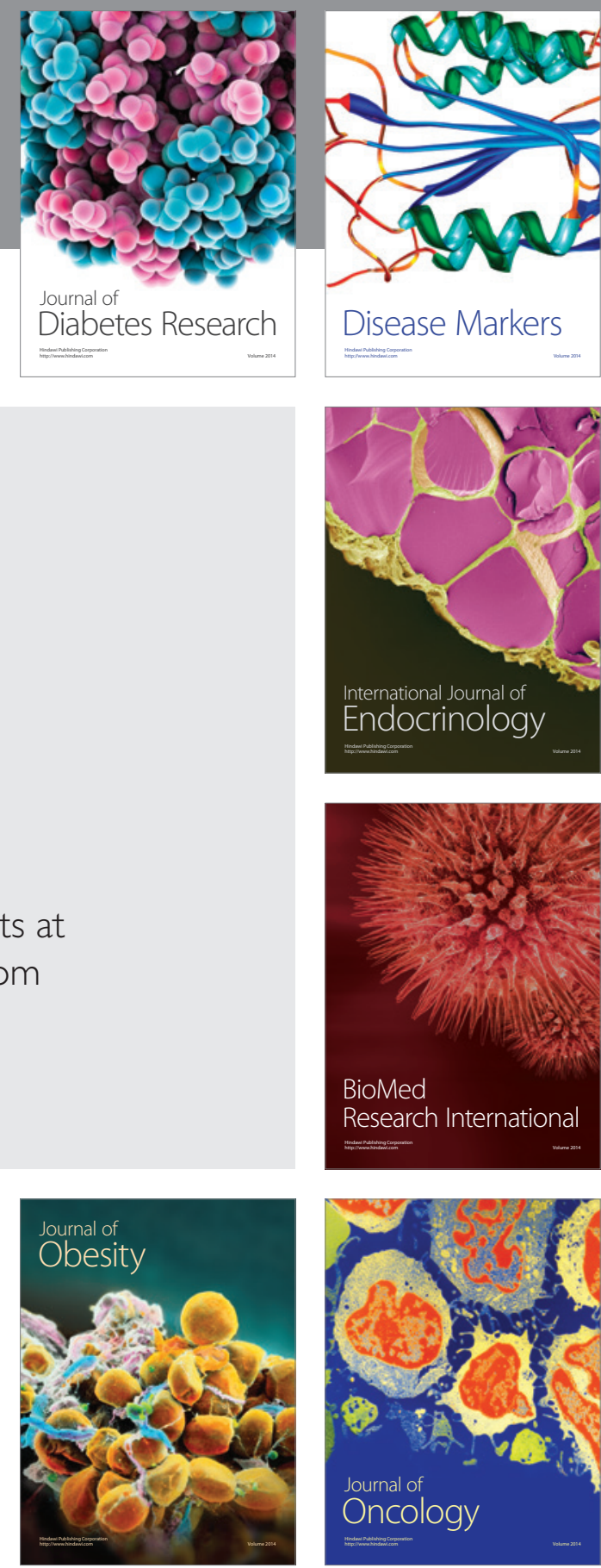

Disease Markers
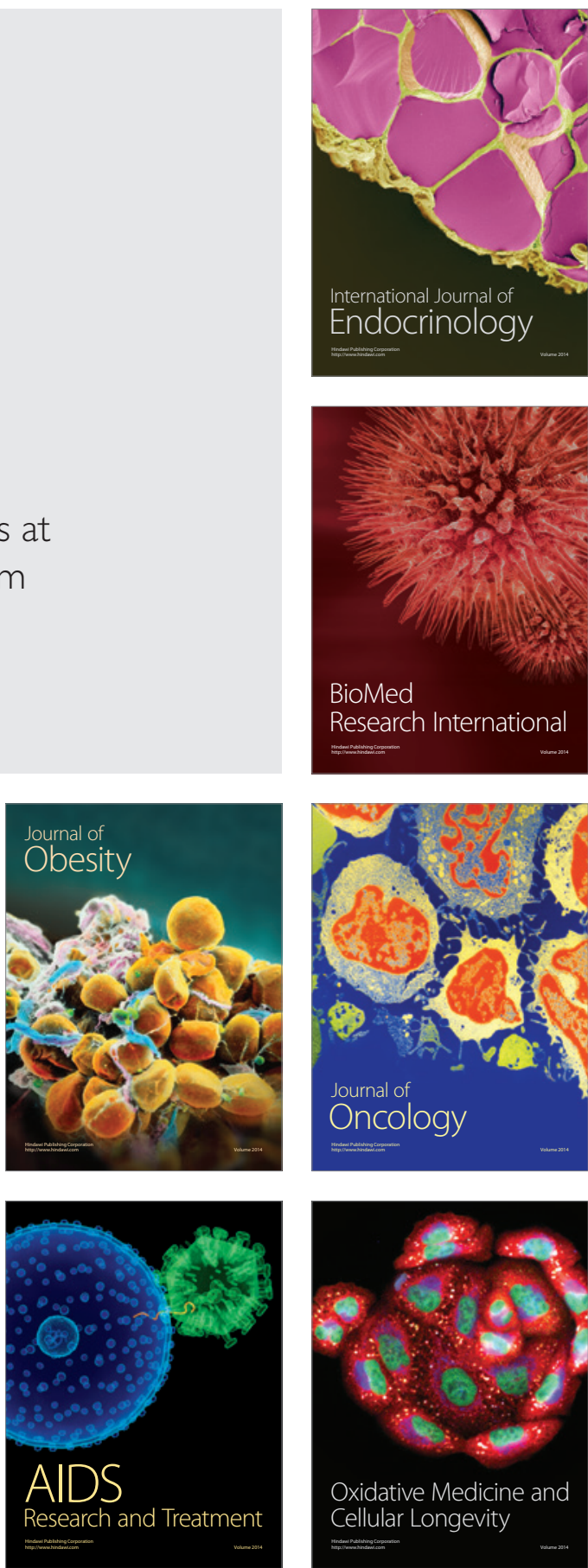